\title{
OPHTHALMIC, CONDITIONS IN RETURNED PRISONERS OF WAR
}

\section{We have received the following note from our Melbourne corres- pondent:-}

The oculists of Sydney and Melbourne have been welcoming their P.O.W. colleagues back after $3 \frac{1}{2}$ years in Singapore. They are Dr, F. P. Chaffy of Sydney and Dr. Robin Orr of Melbourne. After conversations with Dr. Orr, a friend sent the following notes :-

Following the poor dietary given us by the I.J.A. in 1942 following capitulation, deficiency diseases became rife including diseases of the eye. A corneal lesion occurred first and this was closely followed by an outbreak of retrobulbar neuritis-very similar to tobacco amblyopia.

Gradually a measure of control over the trouble was obtained, some research work being attempted to elucidate the condition and its treatment. Such treatment as was available appeared to be satisfactory particularly if the cases were recognised in the earliest stages. For this purpose a clinic was established which at the busiest period was dealing with 30 , new patients daily and about 140-150 reviews; so as to detect any deterioration in those complaining of failing vision. These were reviewed weekly or at most fortnightly, and if deterioration had set in, they were hospitalised in addition to such treatment (rice polishings, yeast and marmite in small quantity) as was available. Of the A.I.F. about 2,000 were affected (about 15,000 troops were originally in the camp), by retrobulbar neuritis, and about 800 by the corneal lesion.' The corneal lesion cleared up and left no disability, but the retrobulbar neuritis in many cases has left a more or less disabling amblyopia. The lesion is typically bilateral. Many Dutch and English cases were also seen and treated.

It is hoped that some light may be thrown upon the pathology of the condition by Major Maynard who has brought home several complete brain sections with the optic tract from the eye to the occipital cortex, for sectioning and staining under proper conditions.

It was realised that the diseases were probably due to nutritional deficiencies, an the vitamin B complex was considered in this respect. When administered in the form of marmite, fair results were obtained. . But it became necessary to supplement our stocks and so other substances had to be sought. Rice polishings were tried and were not satisfactory, in spite of good quality. Yeast was made and apparently gave some help. But from evidence obtained it seemed that riboflavin was one of the important deficiencies and this was known to be present in green leaves and grass.

Experiments were carried out with leaf extract and proved to be 
satisfactory. Later on grass was substituted owing to difficulty in getting sufficient leaves. Soya beans were also used, when available and a preparation of "Tempi" made by them with a mould and then frying or baking them into a quite palatable cake. By these various means the diseases were apparently controlled and in some cases completely cured.

\section{Memorandum on the Notification and Certification of the Blind,} on Definitions of Blindness, and on Blind Pensions

The following memorandum on notification and certification of the Blind has been drawn up by the Institute of Ophthalmology, Royal Eye Hospital.

More perhaps than any other group of physically handicapped persons, the blind are ensured of public sympathy and help. In 1942 there were 75,306 registered blind persons in England and Wales-far too many for 'unco-ordinated benevolence. Indeed indiscriminate public sympathy is largely responsible for such a problem as that of blind mendicancy. The blind long to feel themselves useful citizens with duties as well as rights. It should be the aim of those interested in blind welfare not only to prevent physical want, but to lessen the sense of uselessness of which blind persons so frequently complain. There are already large numbers of happy and independent blind workers, self-respecting, often self-supporting, performing tasks useful to the community. The promotion of such an economic status should be the ideal of the welfare worker.

Chief among the obstacles to this is advanced age. In 1942 76 per cent. of the registered blind were over 50 years of age. Many had additional disabilities such as deafness, mental deficiency, or deformity and crippling. Blind workers have many expenses not incurred by sighted workers. Prospective employers tend to shun blind workers, lest they should have a greater liability to accident, though actuarial investigations prove this supposition to be incorrect. Finally, in pre-war years there existed the problem of National unemployment. If jobs are not available for the fit, disabled persons and the blind are not encouraged in the labour market. It is not surprising that about 80 per cent. of blind were in 1936 classed as unemployable. It is the task of statesmanship to reduce this figure.

1. Statistics on blindness.-Apart from the difficulty in the definition of blindness it seems that no final estimate of the number of blind in the country is possible. In the past information was 